\title{
$A b$ initio study of the structural, electronic, magnetic, and optical properties of silicene nanoribbons
}

\author{
Matteo Ferri, ${ }^{1,2}$ Guido Fratesi, ${ }^{2}$ Giovanni Onida, ${ }^{2}$ and Alberto Debernardi ${ }^{1, *}$ \\ ${ }^{1}$ CNR-IMM, sede Agrate Brianza, via Olivetti 2, I-20864 Agrate Brianza, Italy \\ ${ }^{2}$ ETSF and Dipartimento di Fisica, Università degli Studi di Milano, via Celoria 16, I-20133 Milano, Italy
}

(Received 13 December 2018; published 11 February 2019)

\begin{abstract}
We present first-principles calculations of structural, electronic, magnetic, and optical properties of zigzagoriented silicene nanoribbons, which, being endowed with spin-polarized edge states, are promising candidates as building blocks of future spintronic devices. The minimal width for a structurally stable planar structure having zigzag edges corresponds to a 4-chain ribbon, whose ground state presents antiferromagnetically coupled spin-polarized edges, and a lattice parameter along the nanoribbon axis contracted $(\sim 5 \%)$ with respect to the bulk value. Starting from the dependence of structural and electronic properties on the ribbon width, we present theoretical predictions for the optical spectra of narrow nanoribbons, in which excitonic effects are relevant due to the confinement in a quasi-one-dimensional structure. Especially for light polarized parallel to the ribbon axis, we find significant differences in the position of optical absorption peaks of ribbons with ferroor antiferromagnetically coupled edges, showing that optical spectra can be used as a fingerprint of the magnetic coupling of electronic edge states.
\end{abstract}

DOI: 10.1103/PhysRevB.99.085414

\section{INTRODUCTION}

Graphene, a single layer of carbon atoms packed into a two-dimensional (2D) honeycomb lattice, has attracted considerable attention in the scientific community for its peculiar properties since a method to produce freestanding flakes by mechanical exfoliation was proposed in 2004 [1]. This discovery paved the way to the engineering of novel 2D graphene-like materials. Silicene, the analog of graphene based on silicon, can take advantage of its compatibility with the current industrial processes based on the silicon technology. The traditional scaling of the electronic devices had reached limitations of electrostatics and short-channel effects, threatening the continuance of Moore's law. The industry has already moved to thin-film channel devices, and a Si-based 2D material such as silicene is a good candidate to reach the ultimate limit of channel thickness [2]. A 2D structure of silicon was predicted a decade before the advent of graphene by first-principles total-energy calculations [3]. The most important achievement obtained in that pioneering work was that the equilibrium geometry of $2 \mathrm{D}$ silicon is a corrugated structure, favored with respect to the planar one. Cahangirov et al. predicted, by means of a first-principles structural optimization and phonon dispersion, that silicene can be stable in a low-buckled honeycomb structure, due to a mixed $s p^{2}-s p^{3}$ hybridization [4]. They also predicted that the low-buckled silicene is a semimetal and its bands cross at the Fermi level forming a Dirac cone centered at the $\mathrm{K}$ point in the Brillouin zone, similarly to graphene. As a consequence, the charge carriers resemble massless Dirac fermions. Furthermore, it has been recently predicted that the realization of silicene-germanene $2 \mathrm{D}$ heterostructures could allow one to

\footnotetext{
*alberto.debernardi@mdm.imm.cnr.it
}

engineer the band structure and the Fermi velocities within the Dirac cone [5].

Nevertheless, no successful attempt to synthesize freestanding silicene has been reported. Recently Du et al. obtained a quasi-freestanding layer through the oxidation of bilayer silicene on the $\operatorname{Ag}(111)$ surface [6]. Silicene has been grown on various metallic substrates, such as $\mathrm{Ag}[7,8], \mathrm{ZrB}_{2}$ [9], and Ir [10]. The most frequently investigated phase is a $4 \times 4$ silicene reconstruction on the $\operatorname{Ag}(111)$ substrate [7]. However, a strong hybridization between the silicene states and the silver ones affects both the electronic $[11,12]$ and the optical properties [13].

This strong coupling between the epitaxial silicene lattice and its metal-hosting substrate inhibits the required semiconducting properties and represents a drawback in the fabrication of functional electronic devices [14]. Nevertheless, the reported electrical decoupling of silicene from its silver substrate and the announced fabrication of the first functional transistor device with a transferred silicene layer on insulating substrates [15] indicate that the problem of the metallic substrates could be overcome.

The passivation of the dangling bonds of silicene with adatoms, such as $\mathrm{H}$, that yields silicane, the silicon analog of graphane, is a possible way to produce a gap and to form a $2 \mathrm{D}$ semiconductor [16]. An alternative route to achieve the opening of the energy gap in silicene is to restrict the geometry to one dimension, by constructing nanoribbons, i.e., silicene stripes of finite width. The honeycomb structure of silicene allows the formation of mainly two kinds of edges, namely the armchair and the zigzag ones, defined by the relative orientation of the hexagons relative to the ribbon length. First-principles calculations predicted that freestanding silicene nanoribbons with armchair edges are nonmagnetic [4], while zigzag edges are magnetic [4,17]; in particular, zigzag edges have an antiferromagnetic semiconducting ground state, 
which may find some possible applications in nanoelectronics and spintronics [17]. Up to now, silicene has been synthesized in the form of nanoribbons on the anisotropic $\mathrm{Ag}(110)$ surface [18], showing a metallic character [19] and a welldefined width of integer multiples of $0.8 \mathrm{~nm}$ [20]. However, the atomic structure of the nanoribbons remains unknown. There is a complex interplay between the amount of deposited silicon and the deposition temperature, resulting in a rich array of possible self-assembled nanostructures and surface reconstructions [20]. Several reconstruction models have been proposed, both for freestanding silicene nanoribbons [21,22] and for silicene nanoribbons on Ag [23].

The aim of this work is to provide an ab initio calculation of the optical properties of freestanding zigzag oriented silicene nanoribbons focusing, in particular, on the role played by the inclusion of quasiparticle and electron-hole corrections. We show in the present work that the optical spectrum is a powerful tool to characterize structural and magnetic properties of ribbon edges. The dependence of band gaps and absorption spectra peaks on the nanoribbon width allows a tuning of magneto-optical properties, which can be modified according to the needs of the electronics industry.

We have simulated zigzag Si nanoribbons (ZSiNRs), for their potential application in forthcoming spintronic devices; further, we focus our study on narrow nanoribbons, where confinement effects are expected to be larger. After a brief overview of methods and technicalities used in the present work (Sec. II), we will present our results for structural (Sec. III), electronic (Sec. IV), and optical (Sec. V) properties of ZSiNRs.

\section{COMPUTATIONAL METHODS}

We performed our structural and electronic calculations in the framework of density functional theory (DFT), solving the Kohn-Sham (KS) equations through the plane-wave pseudopotential approach, as implemented in the Quantum Espresso (QE) open-source code [24]. Following our previous work [25], we chose a fully relativistic norm-conserving pseudopotential, generated with a Perdew-Burke-Ernzerhof (PBE) exchange-correlation functional [26], and a 75 Ry cutoff radius for the wave function. Adopting the standard convention, the width of the nanoribbon is classified by the number of zigzag chains across it. So we label a nanoribbon with $N_{z}$ zigzag chains as $N_{z}$-ZSiNR. We described the geometric structure of the ribbon using the supercell method with periodic boundary conditions, adopting a tetragonal cell which we double along the ribbon direction in order to reproduce the $(2 \times 1)$ edge reconstruction reported in the literature [4,21]. We set a vacuum region of $15 \AA$ in the directions perpendicular to the ribbon axis in order to avoid spurious interactions between the periodically repeated replicas of the ribbon. The Brillouin zone (BZ) is sampled with a $45 \times 1 \times 1$ Monkhorst-Pack grid [27] to properly describe the Fermi surface in the nonmagnetic (NM) and in the ferromagnetic (FM) configuration. In the semiconducting antiferromagnetic configuration (AFM) we sampled the BZ with a less dense $15 \times 1 \times 1$ MonkhorstPack grid, since we have verified that the error on the total energy is already lower than $10^{-3} \mathrm{meV} /$ atom in this case. The atomic positions and the lattice constant are fully relaxed by a Broyden-Fletcher-Goldfarb-Shanno (BFGS) algorithm [28] with a threshold on the forces of $0.25 \mathrm{meV} / \AA$.

The optical calculations were carried out using the Yambo package [29]. The macroscopic dielectric constant was computed using 230 empty states (spanning an energy region up to $8 \mathrm{eV}$ above the Fermi level), and a $25(60) \times 1 \times 1 \mathbf{k}$-point mesh for the AFM (FM) ribbons. The effect of the local fields has been taken into account with an energy cutoff of 1 Ry and 2 Ry for the FM and the AFM configurations, respectively. Those values differ because the greater number of $\mathbf{k}$ points used to sample the BZ of the FM 4-ZSiNR requires more computational resources forcing us to limit the values of the other parameters. We checked for the AFM case that this reduced computational setup does not alter the conclusions of the present work. We solved the Bethe-Salpeter equation (BSE) within the Tamm-Dancoff approximation in order to include the excitonic effects. The two-particle Hamiltonian has been constructed with 24 occupied and 24 empty states for the AFM configuration and with 10 occupied and 10 empty for the FM case.

\section{STRUCTURAL PROPERTIES}

We constructed ZSiNRs cutting stripes of different widths from the geometric structure of the low-buckled honeycomb silicene. We found two different edge reconstructions after the structural optimization, which are shown in Fig. 1. In both of them one Si atom at the edge is pushed down, while the adjacent atom is raised. This situation is emphasized especially in Fig. 1(b), in which one Si atom gets close enough to an inner Si atom to induce it to form a new bond, making a triangle-pentagon pair. These atomic arrangements were studied for the first time by Li et al. [21], who have shown that

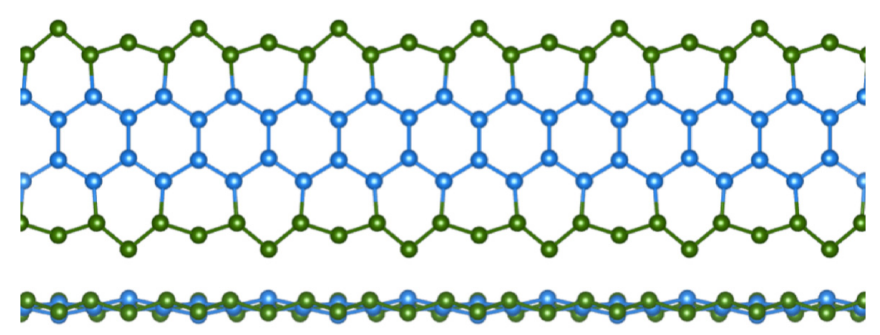

(a)

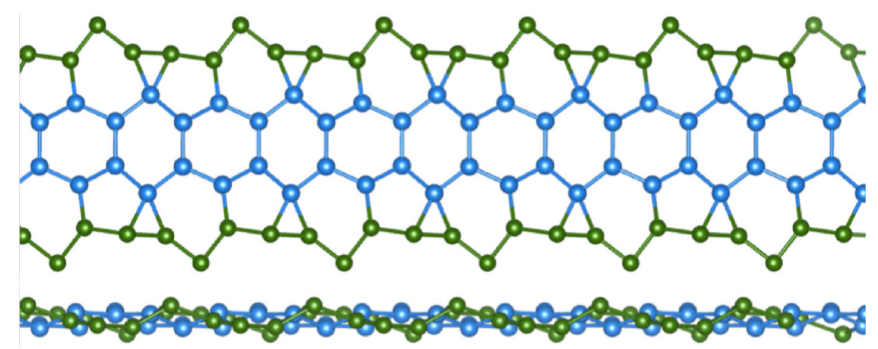

(b)

FIG. 1. Top and side views of reconstructed structures of 4-chain silicene nanoribbons having zigzag edges. The structure (b) has energy $28 \mathrm{meV} \AA^{-1}$ lower than the structure (a). Edge atoms have a different color from the bulk ones to increase readability. 

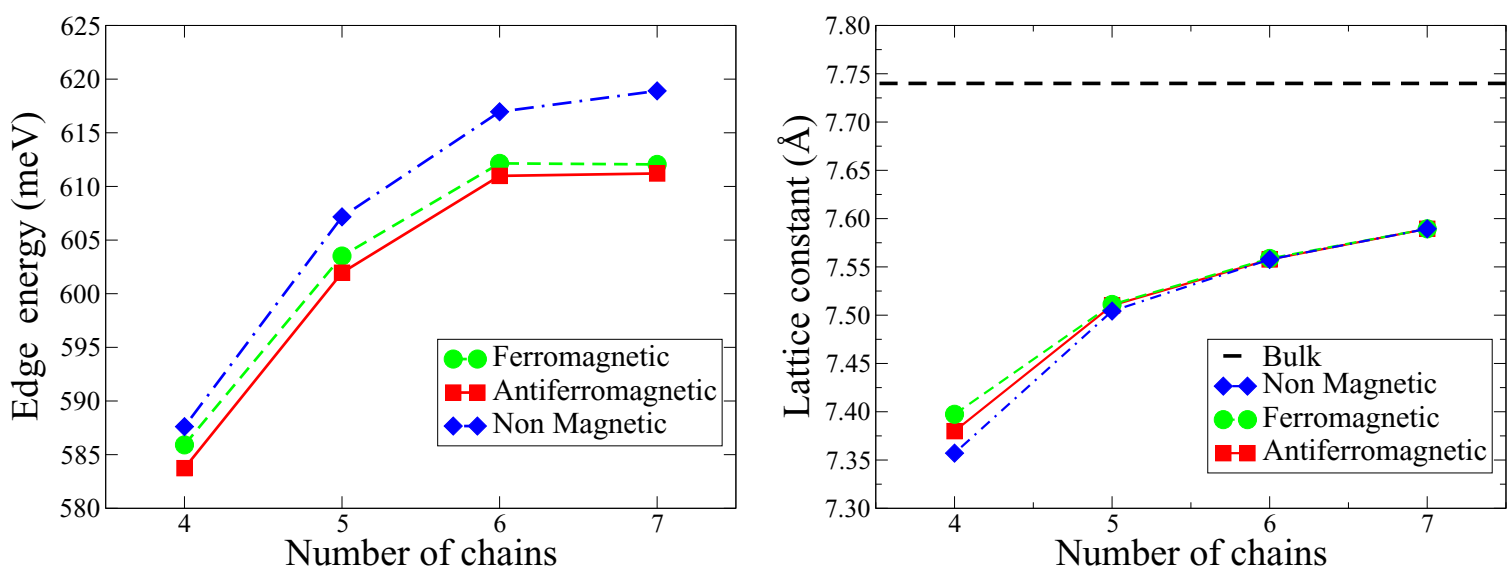

FIG. 2. Left panel: Edge energy per atom in the edge chain for different width of the nanoribbon for antiferromagnetic (squares), ferromagnetic (circles), and nonmagnetic (diamonds) spin polarization at ribbon edges. Right panel: Lattice constant for different width of the nanoribbon for antiferromagnetic (squares), ferromagnetic (circles), and nonmagnetic (diamonds) spin polarization at ribbon edges. Lines are a guide to the eye.

the structure with the triangle-pentagon pair defect is the most energetically favored one. The formation of a new covalent bond contributes to lowering the edge energy, defined as

$$
E_{\text {edge }}=\frac{E_{\text {ribbon }}-N \times E_{\mathrm{Si}}}{2 a},
$$

where $E_{\text {ribbon }}$ represents the total energy per unit cell of the ribbon, $N$ is the number of atoms within the unit cell, $E_{\mathrm{Si}}$ the energy per atom in an infinite layer of silicene, and $a$ the lattice constant of the ribbon (parallel to the ribbon axis). We found that the edge energy of the triangle-pentagon configuration is $28 \mathrm{meV} \AA^{-1}$ lower than the one of the structure depicted in Fig. 1(a), in agreement with the values obtained in Ref. [21].

It is known from the literature that ZSiNRs have an antiferromagnetic ground state [17,21], similarly to graphene zigzag nanoribbons [30]. Therefore we investigated how the inclusion of the spin polarization affects the stability and the structural parameters of this peculiar atomic arrangement. Two different kinds of spin-polarized structures are taken into account: a configuration with an opposite spin orientation between two ferromagnetically ordered edges [conventionally called antiferromagnetic configuration (AFM)], and its ferromagnetic (FM) counterpart.

We calculated the edge energy per edge atom (i.e., $E_{\text {edge }} \times$ $2 a / N_{\text {edge }}$, where $N_{\text {edge }}$ is the number of edge atoms), for both the AFM and FM configurations, and we compared their value with the one of the NM structure for different widths of the ribbon. The AFM state corresponds to the ground state as shown in the left panel of Fig. 2. The energy difference between the magnetic (AFM, FM) and NM configuration slightly increases with the ribbon width, confirming that a spin polarization of the edge atoms is energetically favored also in the limit of a semi-infinite 2D silicene sheet. At variance, the energy difference between the AFM and FM configuration decreases as the ribbon width increases, due to the decoupling between the two magnetic edges.

We considered zigzag nanoribbons of different widths ( $N_{z}$-ZSiNRs) with $N_{z}=4,5,6$, and 7 corresponding to the number of zigzag chains (including the edge ones). As shown in Ref. [25], the 3-ZSiNR is unstable in a nanoribbon config- uration, and spontaneously reconstructs in a kind of nanowire structure, whose total energy per atom is $0.225 \mathrm{eV} /$ atom lower than the starting nanoribbon configuration cut from ideal 2D silicene.

The dependence of the equilibrium lattice constant on ribbon width is depicted in the right panel of Fig. 2. The dashed line shows the lattice constant of infinite 2D silicene, which can be considered as a ribbon of infinite width. The lattice constant increases with the number of chains, with our 7 -chain ribbon being still contracted by about $2 \%$ with respect to the bulk limit. The magnetization also has a noticeable effect on the lattice constant, especially in the thinner ribbon (4-ZSiNR, with a difference of about $0.5 \%$ between the lattice constants of ferromagnetic and nonmagnetic structures), since the confinement effects and the edge-edge interaction are stronger and affect the atomic rearrangement. To characterize quantitatively how such atomic rearrangements change the structural properties of the ribbon, we define the buckling of a chain parallel to the ribbon axis as

$$
b_{\text {chain }}=\sum_{i \in \text { chain }}\left|z_{i}-\langle z\rangle\right| \text {, }
$$

where the $z$ axis is taken in the direction perpendicular to the ribbon plane, $z_{i}$ is the coordinate of the position of $i$ th atom along the $z$ axis, and the average $z$ plane is defined as: $\langle z\rangle \equiv$ $\sum_{i \in \text { ribbon }} z_{i}$.

We show in Fig. 3 the $b_{\text {chain }}$ values for atoms placed at the edges $\left[b_{\text {edge }}\right.$; Fig. 3(a)] and for those placed in the central rows of the ribbon [ $b_{\text {center }}$; Fig. 3(b)].

The dashed lines represents the limit for infinite 2D silicene $(0.23 \AA)$, i.e., half the silicene buckling. While the atomic arrangement of the atoms placed at the edges is quite independent of the width of the ribbon, the atoms at the center tend to converge rapidly to the configuration of 2D infinite silicene, consistently with a rapidly decreasing variance (shown by the error bars), as we can see also from the decreasing error bars. 
(a)

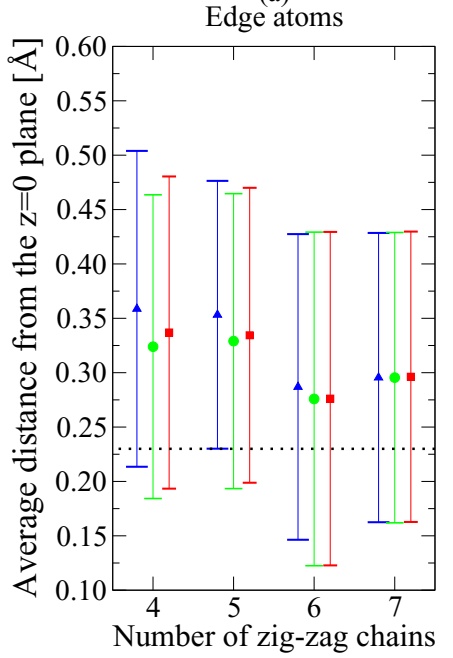

(b)

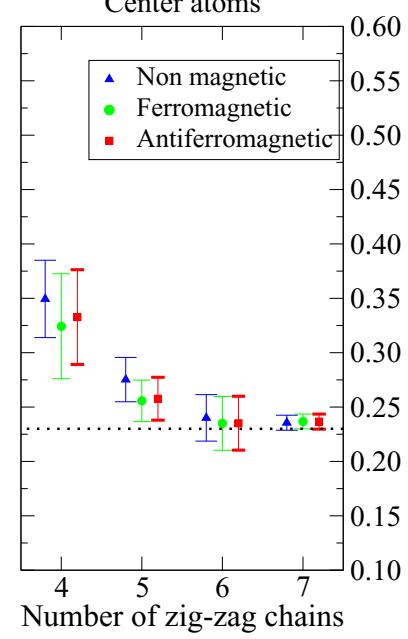

Center atoms

FIG. 3. Atomic buckling for different widths of the nanoribbon (see text). The error bars represent the standard error of the set of the distances of atoms along the (edge/central) chain from the $z=0$ plane. For a given number of chains, the values corresponding to different magnetic structures are slightly displaced along the horizontal axis to increase readability.

\section{ELECTRONIC PROPERTIES}

We focus on narrow nanoribbons in which confinement effects and the magnetic coupling between the two edges are expected to be more relevant than in the case of larger ribbons such as those considered by Li et al. [21].

We display in Fig. 4 our computed band structures and the corresponding electronic density of states (DOS) for the 4-ZSiNR, the shorter width allowed for a structurally stable ZSiNR [25]. We compare the nonmagnetic (NM), antiferromagnetic (AFM), and ferromagnetic (FM) spin-polarized cases. Band structures of larger 5-ZSiNR and 6-ZSiNR are shown in Appendix B. We remark that all these structures
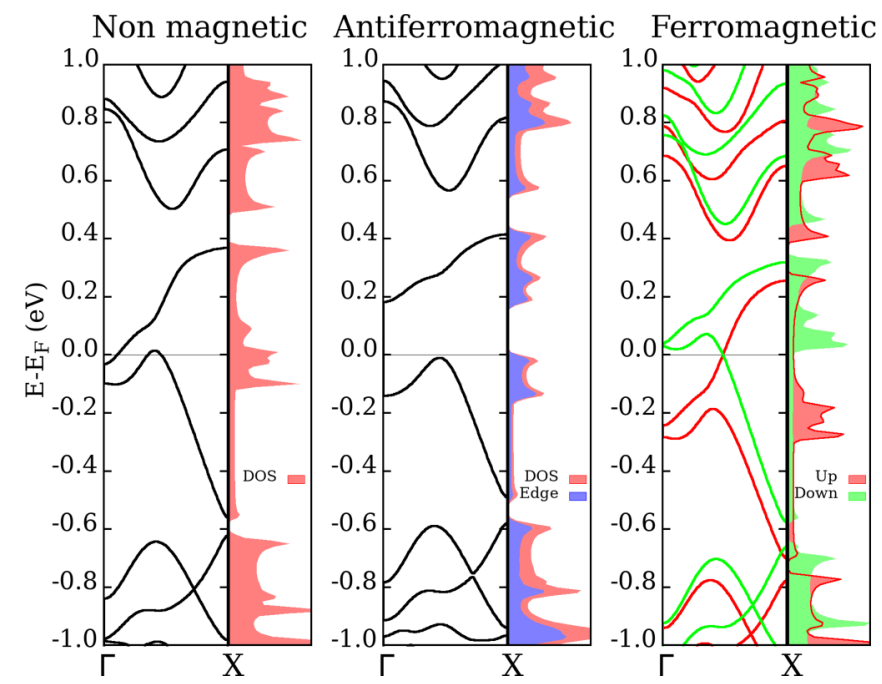

FIG. 4. Electronic band structures of 4-chain zigzag silicene ribbon, for nonmagnetic, antiferromagnetic, and ferromagnetic configuration of spin density at the edges. feature a $(2 \times 1)$ reconstruction of the edges, so they have the same one-dimensional BZ (apart from small changes in the optimized lattice constant).

We first focus our attention on the band structure of the NM (spin-unpolarized) 4-ZSiNR, reported in the left panel of Fig. 4 with the corresponding DOS. This system is metallic with two bands crossing the Fermi level, and a double peak in the DOS close to $E=0$. Comparing with the results of Li et al. [21] for the 8-chain nanoribbon in a nonmagnetic configuration where the two bands close to the Fermi level are almost degenerate in the first half BZ, our results for the 4chain ribbon show them as well separated and distinguishable. This is consistent with a much stronger edge-edge interaction, which increases the level splitting in the narrower ribbon. The double DOS peak near the Fermi level (DOS at $-0.02 \mathrm{eV}$ and at $0.01 \mathrm{eV}$ ) is essentially due to $p_{z}$ electrons of edge atoms, according to our results for the projected density of states (PDOS) on the atomic orbitals [31]. The presence of a sharp double peak in the DOS close to the Fermi level may lead to a magnetic transition [32]. This is consistent with our result showing that the magnetic configurations are energetically favored (see Sec. III).

In the following, we analyze the band structure of the magnetic systems. In all the ZSiNRs studied the lowest-energy magnetic configuration is the one with an AFM coupling between the edges. The ground state of the 4-ZSiNR is hence $\mathrm{AFM}$, and remains so also when structural relaxations are included. The AFM 4-ZSiNR band structure and DOS are reported in the central panel of Fig. 4. The magnetization opens an energy gap, shifting upwards the band immediately above $\mathrm{E}_{F}$, hence removing the double DOS peak at the Fermi level and creating a range of forbidden energies. The system displays a small indirect gap of $0.194 \mathrm{eV}$ between the valence band maximum (VBM) and the conduction band minimum (CBM) at the $\Gamma$ point. This makes the $4-Z S i N R$ a magnetic semiconductor. However, the bands are still twofold spin degenerate, consistently with the general properties of a system with an equal number of spin-up and spin-down electrons with a spatial inversion symmetry operation, together with time-reversal invariance [33].

The uppermost valence band and the first conduction bands are almost parallel in the first half of the BZ, as we can see by comparing the direct gap at the $\Gamma$ point, $0.32 \mathrm{eV}$, with the $0.29 \mathrm{eV}$ direct gap at VBM. We report in our PDOS analysis (Fig. 4, central panel) the projected DOS on the edge atoms only, which shows that bands near the Fermi level are essentially edge states.

The FM 4-ZSiNR is only slightly higher in energy (energy per $\mathrm{Si}$ atom $1.1 \mathrm{meV} /$ atom) than the $\mathrm{AFM}$ one, and presents several interesting features. Its spin-polarized band structure is shown in the right panel of Fig. 4. The exchange splitting of the bands corresponding to spin-up and spin-down electrons is evident, with the largest separation $(0.3 \mathrm{eV})$ displayed by bands close to the Fermi level. The exchange splitting moves two spin-down bands above $\mathrm{E}_{F}$ at the $\Gamma$ point. The lowest of them becomes then occupied around the middle of the BZ, by linearly crossing a spin-up band at the Fermi level. Each spin polarization displays a metallic behavior, but the global band structure is that of a semimetal, because the DOS at the Fermi level is very small for both spin polarizations. 
The net magnetization of the material comes from the different number of spin-up and spin-down occupied states. The band crossing point at the Fermi level occurs at slightly before half of the $\Gamma$-X direction $\left(X / 2=\frac{1}{4} \frac{2 \pi}{a}\right)$ at $k=0.24 \frac{2 \pi}{a}$, so that the spin-up electrons fill almost two half bands more than spin-down ones. Hence the total magnetization is $0.97 \mu_{B} /$ cell (see also Table II in Appendix B), slightly lower than the value of $1 \mu_{B} /$ cell that would have been obtained for a crossing point at $X / 2$. This linear crossing on the Fermi level is particularly interesting because it resembles the shape of the Dirac cone in 2D honeycomb silicene, here for states of different spin. At the $\Gamma$ point, these two bands (the uppermost filled and the lowermost empty bands) are mainly constituted by edge electrons, but this contribution progressively decreases as the wave vector approaches the $\mathrm{X}$ point, a peculiar property discussed in Appendix A, together with other features of the band structure of the FM 4-ZSiNR.

In summary we found that the AFM 4-ZSiNR is a magnetic semiconductor, with a small indirect energy gap of about $0.2 \mathrm{eV}$. The FM 4-ZSiNR is a semimetal with a linear dispersion around the Fermi level, similar to the Dirac cone in $2 \mathrm{D}$ honeycomb silicene. Despite the fact that it shows a somewhat similar behavior to the $2 \mathrm{D}$ silicene, the FM structure represents an excited configuration, since the AFM one is lower in energy. Due to its semiconducting properties, the AFM 4-ZSiNR could be a candidate to be integrated in electronic devices.

\section{OPTICAL PROPERTIES}

The imaginary part of the macroscopic dielectric function, $\epsilon_{M}(\omega)$, is a key property for technological applications because it is proportional to the optical absorption. The $\epsilon_{M}(\omega)$ is related to microscopic quantities which can be computed on the basis of transition matrix elements between electronic states, by considering both interband and intraband transitions in the case of metals.

In our study we considered the long-wavelength limit $(q \simeq 0)$, which is appropriate for spectra obtained by ultraviolet-visible spectroscopy (UV-VIS). In this limit the macroscopic dielectric function $\epsilon_{M}$ reads

$$
\epsilon_{M}(\omega)=\lim _{\mathbf{q} \rightarrow 0} \frac{1}{\left[\epsilon_{\mathbf{G G}^{\prime}}^{-1}(\mathbf{q}, \omega)\right]_{\mathbf{G}=0 \mathbf{G}^{\prime}=0^{\prime}}},
$$

which involves only the term having the reciprocal lattice vectors $\mathbf{G}=0, \mathbf{G}^{\prime}=0$, i.e., the so-called "head" of the inverse microscopic dielectric function written in reciprocal space as $[35,36]$

$$
\epsilon_{\mathbf{G G}^{\prime}}^{-1}(\mathbf{q}, \omega)=\delta_{\mathbf{G G}^{\prime}}+v(\mathbf{q}+\mathbf{G}) \chi_{\mathbf{G G}^{\prime}}(\mathbf{q}, \omega),
$$

where $v$ is the Coulomb potential and $\chi_{\mathbf{G G}^{\prime}}(\mathbf{q}, \omega)$ represents the reducible polarizability, which in the random-phase approximation (RPA) is expressed in terms of the irreducible polarizability as

$$
\begin{aligned}
\chi_{\mathbf{G G}^{\prime}}(\mathbf{q}, \omega)= & \sum_{\mathbf{G}^{\prime \prime}}\left[\delta_{\mathbf{G G}^{\prime \prime}}-v\left(\mathbf{q}+\mathbf{G}^{\prime \prime}\right) \chi_{\mathbf{G G}^{\prime \prime}}^{0}(\mathbf{q}, \omega)\right]^{-1} \\
& \times \chi_{\mathbf{G}^{\prime \prime} \mathbf{G}^{\prime}}^{0}(\mathbf{q}, \omega) .
\end{aligned}
$$

Here, the noninteracting response function $\chi_{\mathbf{G G}^{\prime}}^{0}(\mathbf{q}, \omega)$ is constructed from the single-particle eigenvalues $\left(\varepsilon^{0}\right)$, eigenfunctions $\left(\psi^{0}\right)$, and occupations $(f)$ as

$$
\begin{aligned}
\chi_{\mathbf{G G}^{\prime}}^{0}(\mathbf{q}, \omega)= & -\frac{1}{V} \sum_{n \mathbf{k}} \sum_{m \mathbf{k}^{\prime}}\left(f_{n \mathbf{k}}-f_{m \mathbf{k}^{\prime}}\right) \\
& \times\left(\frac{\left\langle\psi_{m \mathbf{k}^{\prime}}^{0}\left|e^{i(\mathbf{q}+\mathbf{G}) \mathbf{r}}\right| \psi_{n \mathbf{k}}^{0}\right\rangle\left\langle\psi_{n \mathbf{k}}^{0}\left|e^{-i\left(\mathbf{q}+\mathbf{G}^{\prime}\right) \mathbf{r}^{\prime}}\right| \psi_{m \mathbf{k}^{\prime}}^{0}\right\rangle}{\varepsilon_{m \mathbf{k}^{\prime}}^{0}-\varepsilon_{n \mathbf{k}}^{0}-\omega-i \eta}\right),
\end{aligned}
$$

where $V$ is the unit cell volume.

The simplest (and crude) approximation is the independent-particle RPA [35,36], in which the response function is computed by using the Kohn-Sham (KS) eigenvalues $\left(\varepsilon^{0}=\varepsilon^{K S}\right)$ and eigenfunctions $\left(\psi^{0}=\psi^{K S}\right)$, and including only the $\mathbf{G}=0, \mathbf{G}^{\prime}=0$ terms in Eq. (5). The KS-RPA spectrum is obtained by summing explicitly the $\mathbf{G} \neq 0, \mathbf{G}^{\prime} \neq 0$ terms up to the energy cutoff. However, this spectrum is constructed from the DFT Kohn-Sham eigenvalues $\left(\varepsilon^{K S}\right)$ and eigenfunctions $\left(\psi^{K S}\right)$ and it is well known that DFT underestimates the energy gap in semiconductors and insulators and, therefore, also the optical absorption edge. To overcome this problem, we move from the DFT energies to the quasiparticle (QP) energies in the framework of many-body perturbation theory (MBPT). The electronic structure is corrected replacing the DFT exchange-correlation potential with the nonlocal self-energy operator $\Sigma$ :

$$
\varepsilon_{n \mathbf{k}}^{Q P}=\varepsilon_{n \mathbf{k}}^{K S}+\left\langle\psi_{n \mathbf{k}}^{Q P}\left|\Sigma\left(\varepsilon_{n \mathbf{k}}^{Q P}\right)-V_{x c}\right| \psi_{n \mathbf{k}}^{Q P}\right\rangle .
$$

The QP eigenfunctions $\left|\psi_{n \mathbf{k}}^{Q P}\right\rangle$ are taken as the unperturbed Kohn-Sham states $\left|\psi_{n \mathbf{k}}^{K S}\right\rangle$, while the effect of the self-energy can be determined, for example, by the $G W$ approximation [34]. In many cases, a rigid shift of the conduction bands ("scissors operator") followed by a band stretching represents a good approximation to the true corrections, implemented in the Yambo code [29]. Hence we followed this simpler approach, with parameters for the shift and stretching obtained by our $G W$ calculations for a narrower set of bands. We refer to Ref. [31] for further details.

The QP response function can be expressed in terms of single-particle electronic eigenstates by substituting $\left|\psi_{n \mathbf{k}}^{0}\right\rangle=$ $\left|\psi_{n \mathbf{k}}^{Q P}\right\rangle$ and eigenenergies $\varepsilon_{n \mathbf{k}}^{0}=\varepsilon_{n \mathbf{k}}^{Q P}$ in Eq. (6).

The above scheme, however, still neglects the electronhole interaction effects, which, due to the strong confinement, are expected to be important in narrow ribbons. In order to include such effects in an ab initio calculation of optical spectra, we adopted the Bethe-Salpeter equation (BSE) scheme $[35,36]$, as implemented in the Yambo code, to compute the optical spectra at the most refined level.

In the VIS-UV range small-width ZSiNRs are essentially transparent to light polarized perpendicularly to the axis of the ZSiNR, because of the local-field effects (see Ref. [25] for a detailed discussion), which induce a strong quenching of the optical absorption for such polarization. Hereafter, we hence focus our study on the VIS-UV optical response for light polarized parallel to the ribbon axis. 


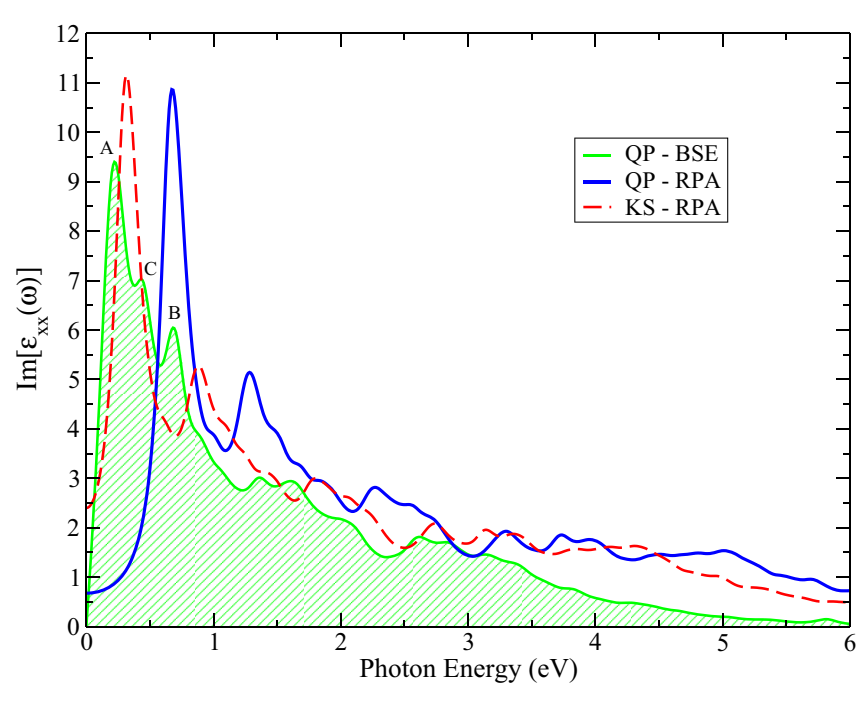

FIG. 5. Optical response of the AFM 4-ZSiNR with electronhole interaction (dashed area) in comparison with the spectra obtained at KS-RPA level (dashed line, from Ref. [25]) and QP-RPA level (blue line).

\section{A. 4-chain antiferromagnetic nanoribbons}

In Fig. 5 we display the imaginary part of the dielectric function for light polarization parallel to the axis of the AFM 4-ZSiNR, for a photon energy spanning the range between 0 and $6 \mathrm{eV}$. The red dashed lines represent the KS-RPA results from Ref. [25], the blue lines represent the QP-RPA ones (in which we applied a rigid upward shift of the empty states of $0.35 \mathrm{eV}$ (obtained from a linear fit of QP corrections of the electronic bands close to the Fermi energy) [31], and the green lines are used for optical spectra obtained by solving the QP-BSE within the Tamm-Dancoff approximation. In the left panel of Fig. 6 we display the QP-corrected electronic bands of the AFM 4-ZSiNR.

The QP-RPA spectrum is characterized by a main absorption peak at $0.7 \mathrm{eV}$, which is due to the transitions between states around the Fermi level in the first half BZ (see Fig. 6, left panel). The energy difference between these weakly dispersing bands is exactly $0.7 \mathrm{eV}$. We notice also a weaker absorption peak at $1.3 \mathrm{eV}$. The band shift due to QP corrections leads to a blueshift of the absorption spectrum with respect to the KS-RPA one.

The inclusion of the electron-hole interaction redshifts the absorption spectrum, giving rise to excitonic states within the QP energy gap. As often found in confined systems, the strongest absorption peak is close in energy to that obtained within the bare KS-RPA. This compensation between selfenergy and excitonic effects is due to balancing between (1) the renormalization of the energy gap due to QP effect, which corrects the DFT-KS gap underestimation producing a blueshift of the spectrum with respect to the KS-RPA results; (2) the electron-hole interaction, which introduces bound states in the electronic band gap and modifies the oscillator strengths, resulting in a redshift of the spectrum with respect to the QP results. Such a compensation is similar to that observed by Bruno et al. in a first-principles study on excitons in $1 \mathrm{D}$ germanium nanowires [37].
The three main absorption peaks of our BSE spectrum for the 4-ZSiNR are labeled as A, B, and C in Fig. 5. The main contribution to the peaks labeled as $\mathrm{A}$ and $\mathrm{C}$ comes from optical transitions between the uppermost valence band and the first conduction band localized in the center of the BZ, as depicted in the left panel of Fig. 6. In this figure, the transitions depicted in red contribute both to peak $\mathrm{A}$ and peak $\mathrm{C}$. The peak $\mathrm{B}$, instead, is due to transitions between the last valence band and the second conduction band (depicted by blue lines in the left panel of Fig. 6).

The exciton binding energy is defined as $E_{b}=\Delta \varepsilon-E_{\text {exc }}$ where $\Delta \varepsilon$ is the difference between the QP energies of the states involved by the transition and $E_{\text {exc }}$ is the excitation energy. The binding energies for these excitons are $0.47,0.42$, and $0.55 \mathrm{eV}$ for the $\mathrm{A}, \mathrm{B}$, and $\mathrm{C}$ peaks, respectively. They are smaller than those found by Prezzi et al. in graphene nanoribbons [38].

We evaluate now the spatial correlation between the electron and the hole. The strongest absorption peak involves transitions from the top of the valence band and in Sec. III we have shown that the edge states provide the main contribution to the uppermost valence band. Therefore we place a hole at the edge and plot the probability distribution of the electron position, corresponding to the lowest excitonic state (A). As shown in the right panel of Fig. 6 (top structure), the electrons tend to localize on both edges, while in the center of the ribbon the probability is lower. This indicates a strong coupling between the edges due to electron-hole interaction. To see the position in which the electron is mostly localized we plot, in Fig. 6 (right panel, bottom structure) a more restrictive isosurface ( $20 \%$ of the maximum square modulus of the electron-hole wave function), showing the edge atoms where the probability to find the electron in a binding excitonic state is greater. The average distance between the electron and the hole was evaluated by computing the distance between the hole and the sites where the electron is mostly localized (see Fig. 6, right panels) and it was estimated to be around $10 \AA$, indicating that the exciton is a kind of 1D Wannier-like electron.

\section{B. 4-chain ferromagnetic nanoribbons}

In Fig. 7 we plot the imaginary part of the dielectric function for light polarization parallel to the axis of the FM 4-ZSiNR (same notation as in Fig. 5).

Since the FM 4-ZSiNR is semimetallic, the intraband transitions should be taken into account in the calculation of the response function. The simplest way to obtain this contribution is to add a semiempirical Drude term, $\epsilon_{D}(\omega)=$ $1-\frac{\omega_{D}^{2}}{\omega\left(i \gamma_{D}+\omega\right)}$, in the dielectric constant. We set the plasma frequency $\omega_{D}=2.3 \mathrm{eV}$ [39], while the damping parameter $\gamma_{D}$ was set to $0.5 \mathrm{eV}$. As for the $\mathrm{QP}$ correction, for this metallic case only a band stretching was required (i.e., without shifting the empty states). We notice that the tendency toward a compensation between the self-energy and the excitonic effects displayed in the AFM spectra is strongly reduced for the FM one, due to the more efficient screening of the latter metallic system. The inclusion of the Drude term does not significantly broaden the peak structure still present at the KS-RPA and QP-RPA levels. 


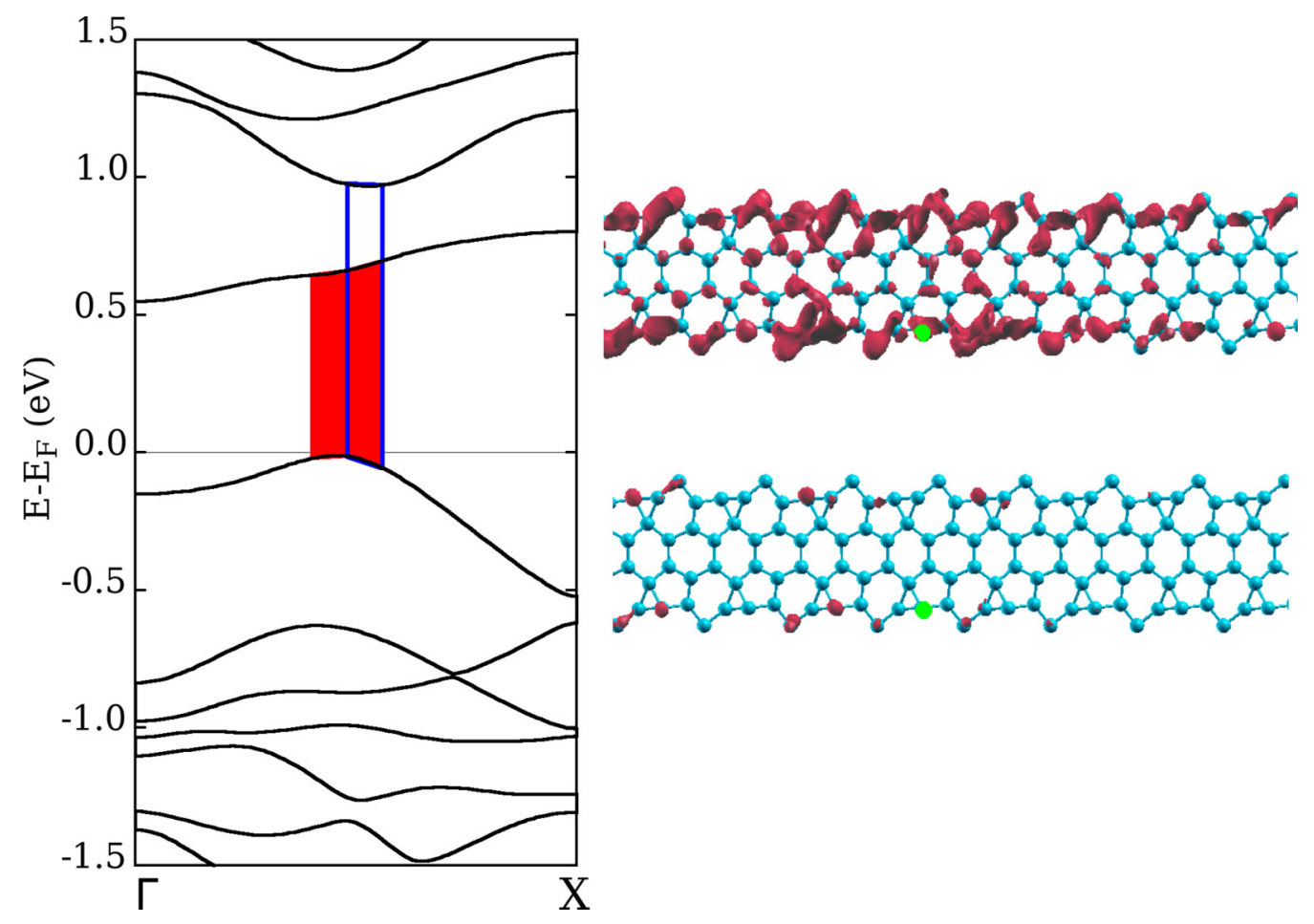

FIG. 6. Left panel: Transitions which mainly contribute to the exciton peaks A and C (red) and B (blue) in Fig. 5. The electronic band structure is corrected with QP self-energies. Right panel: Spatial distribution of the electron for a hole (green dot) fixed at the edge of the ribbon for exciton peak $\mathrm{A}$. The isosurface represents the $2 \%$ (top panel) and the $20 \%$ (bottom panel) of the maximum square modulus of the electron-hole wave function.

The main difference in BSE spectrum with respect to RPA structures is the absence of a broad peak at zero frequency, which can be attributed, at least in part, to the dimensional effect in 1D metallic systems when the electron-hole interaction is taken into account [40].

The BSE has a strong absorption peak at about $0.8 \mathrm{eV}$. The RPA spectra (with the exception of the Drude peak) has a peak structure similar to that of the BSE, but the latter is redshifted

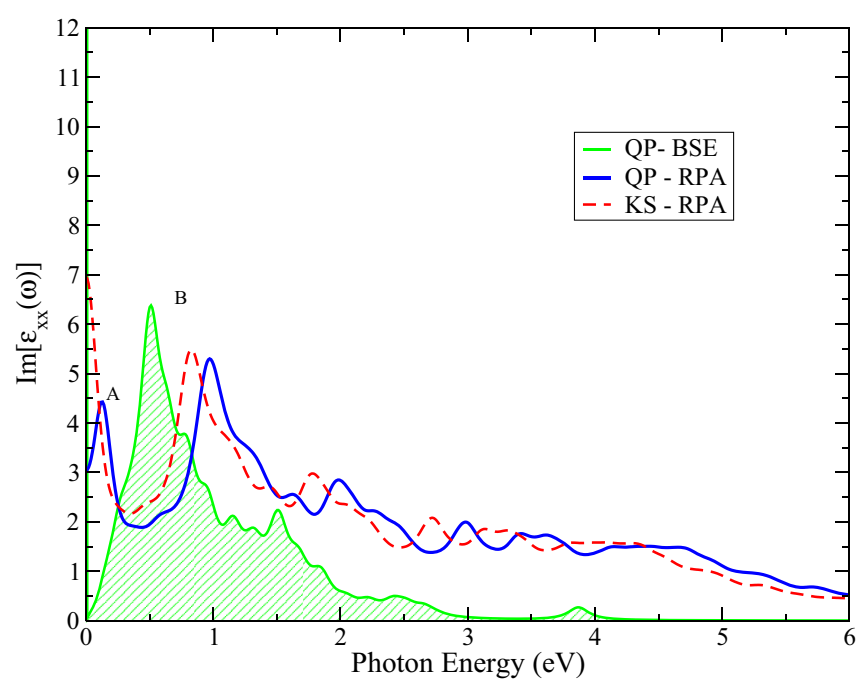

FIG. 7. Optical response of the FM 4-ZSiNR with electron-hole interaction (dashed area) in comparison with the spectra obtained at KS-RPA level (dashed line, from Ref. [25]) and QP-RPA level (blue line). The Drude term has been included. with respect to the former due to electron-hole interaction, with a larger shift than in the former AFM case due to smaller compensation between QP and excitonic effects.

All spectra present a peak at about $0.8 \mathrm{eV}$, while the absorption decreases as the photon frequency increases. The drop of signal computed by the BSE for the FM 4-ZSiNR at frequencies larger than $3 \mathrm{eV}$ is probably due to the limited range of conduction bands we were able to include in the computation of the BSE, as a result of the large computational effort required by the simulation of this system.

The significant difference between the AFM and FM cases in the absorption spectra of 4-ZSiNRs may allow a simple way to discriminate the AFM or FM coupling between the edge states on the basis of optical absorption measurements.

\section{Zigzag nanoribbon of larger widths}

To enlighten the effect of confinement we computed the imaginary part of the dielectric function for light polarization parallel to the axis of the 5-ZSiNR. Our results for the ground state having AFM coupled edges are displayed in Fig. 8. The red dashed lines represent the KS-RPA curves, the blue lines represent the QP-RPA ones, while the green lines represent the optical spectrum obtained by solving the BSE.

The A and C peaks present in the spectrum of the AFM 4-ZSiNR now collapsed in one broadened peak at $\sim 0.35 \mathrm{eV}$. This collapse can be explained by noticing that, in the region of the BZ close to the VBM, the first conduction band of the 5ZSiNR is flatter than the corresponding band of the 4-ZSiNR (see Fig. 10). Since these transitions mainly contribute to peaks $\mathrm{A}$ and $\mathrm{C}$ of the 4-ZSiNR spectra, in the 5-ZSiNR case, 


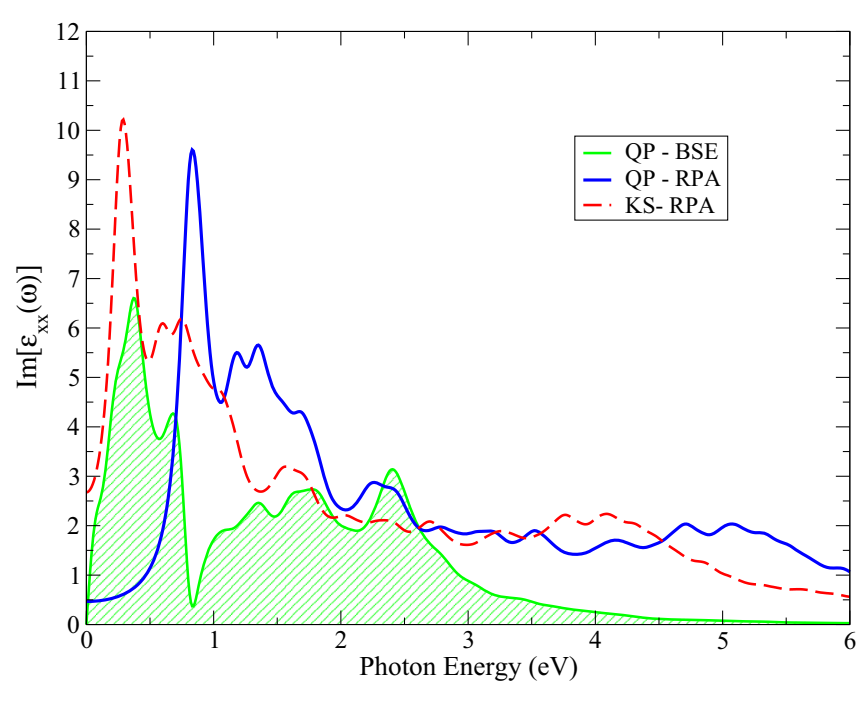

FIG. 8. Optical response of the AFM 5-ZSiNR with electronhole interaction (dashed area) in comparison with the spectra obtained at KS-RPA level (dashed line) and QP-RPA level (blue line).

in the region of the BZ, close to VBM the energy difference between the bottom conduction band and the top valence band is almost constant, thus producing a merging of the $\mathrm{A}$ and $\mathrm{C}$ peaks into a single broader structure.

By comparing the 4-ZSiNR and 5-ZSiNR spectra we notice that the intensity of the main peak (A) decreases as the ribbon width increases. To understand the effects of confinement on the optical properties, we compare our BSE spectra obtained for ZSiNRs with that of 2D honeycomb silicene computed by Wei et al. [42], where the inclusion of the excitonic effects (by the BSE) redshifts the absorption spectrum of about $1 \mathrm{eV}$. In our AFM ZSiNR this value is equal to $0.4 \mathrm{eV}$.

The main effect of the confinement is to remove the peak at higher energies, e.g., the one at $4 \mathrm{eV}$ present in bulk silicene. Further, while the spectrum of bulk 2D honeycomb silicene displays a peak at about $1.3 \mathrm{eV}$, the spectrum of the 5-ZSiNR displays a broadened structure ranging from $1 \mathrm{eV}$ to $3 \mathrm{eV}$.

Significantly, at variance with the AFM 4-ZSiNR case, for the AFM 5-ZSiNR the strong absorption structure due to excitonic peaks in the band gap below $0.8 \mathrm{eV}$ is well separated by the broader structure in the range $1.0 \mathrm{eV}$ to $2.5 \mathrm{eV}$, thus allowing us, at least in principle, to determine with atomic resolution the number of zigzag chains composing the ribbon on the basis of optical spectra.

\section{CONCLUSION}

In summary, we have shown that confinement effects are crucial in short-width zigzag silicene nanoribbons since they significantly modify the lattice parameter, the electronic band structure, the magnetism, and the optical spectra. We investigated the dependence of these properties on the nanoribbon width and on the magnetic coupling of the nanoribbon edges, allowing, at least in principle, the tuning of electronic and magneto-optical properties of the nanoribbons. We studied the result of the inclusion of many-body effects in the optical spectra. In general, the solution of the BSE gives rise to excitonic states which redshift the absorption spectrum, with a partial compensation between the self-energy effects and the excitonic effects. In particular, for the 4-ZSiNR with AFM coupled edges, the strongest exciton peak comes from transitions between the top of the valence band and the lowest conduction band, and the corresponding Wannier-like exciton couples the two edges of the ribbons.

The significant differences in the optical spectra for different ribbon widths or for different magnetic coupling of edge states can be exploited to characterize on the atomic scale the structure and the magnetic configuration of the nanoribbons in future applications in nanoelectronics devices.

\section{ACKNOWLEDGMENTS}

We acknowledge CINECA and the Regione Lombardia award under the LISA initiative for the availability of highperformance computing resources and support [project: $\mathrm{Ab}$ initio Simulation of Silicene nanoribbons for Optoelectronic devices (ASSO)], CINECA for computer resources allocated under ISCRA initiative (project: Optical Properties of Silicene Nanoribbons (OPSIN)], and R. Colnaghi for technical support on computer hardware.

\section{APPENDIX A: ELECTRONIC STRUCTURE OF FERROMAGNETIC 4-ZSiNR}

To investigate the origin of the band crossing of the FM spin-polarized edge structure, we analyze in real space the wave functions corresponding to the bands closest to the Fermi level. In Fig. 9 we report the band structure and wave functions at the $\Gamma$ point, at the crossing point, and at the $\mathrm{X}$ point. At the $\Gamma$ point each wave function is localized at the edges, while, as the wave vector increases, there is a tendency towards combining with states localized at the center of the ribbon. At the $\mathrm{X}$ point we notice that wave functions are mainly localized in the center of the ribbon, for eigenvalues lower than the Fermi energy and along the bonds corresponding to the triangle defect, for the eigenvalues above the Fermi level. It seems that the edge states do not interact with center states at the $\Gamma$ point but they do so as the wave vector increases. Therefore we perform a k-resolved projected DOS (k-PDOS) analysis in order to study the localization of the wave function for each $\mathbf{k}$ point. We project the Bloch function $\left\{\psi_{n, \mathbf{k}}\right\}$ onto a set of atomic orbitals $\left\{\phi_{\bar{n}, l, m}(\tau)\right\}$, where $\tau$ indicates the atomic position within the unit cell and $\bar{n}, l, m$ are the atomic quantum numbers. The k-PDOS is obtained by decoupling the sum over $\mathbf{k}$ from the double summation over $n$ and over $\mathbf{k}$ :

$$
\operatorname{DOS}(E) \simeq \sum_{\mathbf{k}} \sum_{\tau}\left[\sum_{n,(\bar{n}, l, m)}\left|\left\langle\psi_{n, \mathbf{k}} \mid \phi_{\bar{n}, l, m}(\tau)\right\rangle\right|^{2} \delta\left(E-\varepsilon_{n}(\mathbf{k})\right)\right] .
$$

The term in the square brackets is the k-PDOS projection of the atom at $\tau$ which allows us to study the atomic contributions to the band structure. We project the wave function on the edge atoms and on the center atoms for each $\mathbf{k}$ point in the BZ by summing up the term in square bracket on the specific set of atoms at the edge (central) chain. The result is shown in Fig. 9(c). Blue lines indicate a greater localization of the wave 

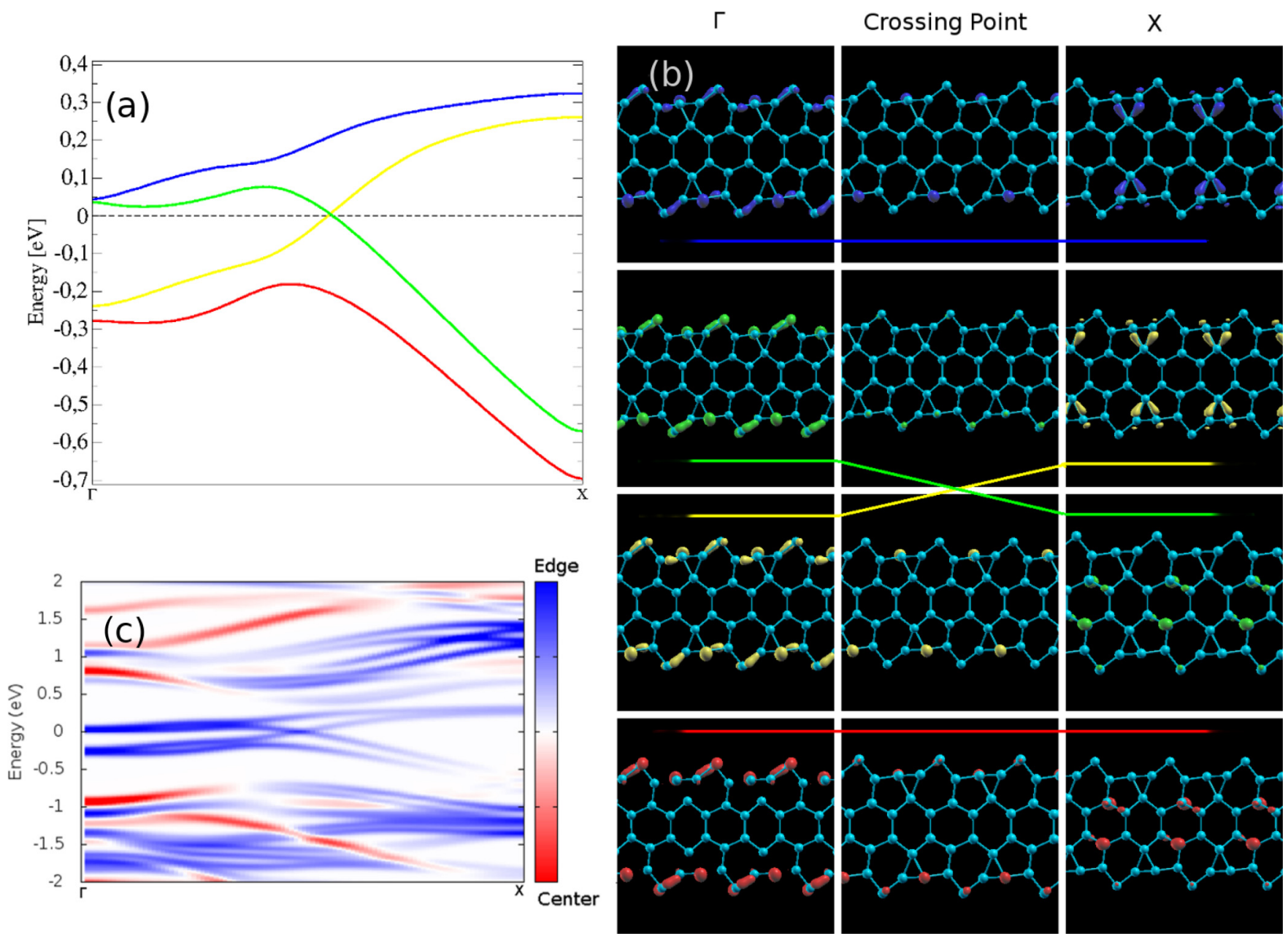

FIG. 9. Ferromagnetic edge states of 4-chain zigzag silicene nanoribbon. Panel (a): Detail of the band structure around the Fermi level. Panel (b): Projection in real space of the wave functions of the bands closest to the Fermi level. From left to right the wave functions reported in real space correspond to the $\Gamma$ point, crossing point, and X point; the isovalue is set to $3 \times 10^{-3}$ (a.u.) ${ }^{-3}$. Panel (c): k-resolved projected density of states. Blue and red lines indicate a greater localization on the edge and the center of the ribbon, respectively.

function on the edge atoms, while red lines indicate a tendency toward localizing on center atoms. White areas indicate that there is an equal contribution, or no states at that energy.

For $\mathbf{k}$ lower than the band crossing value, the bands around the Fermi energy are strongly localized on edge atoms as we can see from the bright blue lines. The first filled/empty states mostly localized on the center atoms are at -0.9 and $0.8 \mathrm{eV}$, respectively. In the second half $\mathrm{BZ}$ the intensity of the blue diminishes, since it approaches the white in correspondence with the valence bands near the $\mathrm{X}$ point. At the $\mathrm{X}$ point there is a slightly higher contribution by atoms of the center but the difference is so small that we cannot distinguish it in this figure. The edge contribution in the conduction bands is lowered but the wave functions are still mainly localized on edge atoms. So the wave function tends towards delocalizing on the whole ribbon as the wave vector increases. We notice some hybrid bands around $\pm 1 \mathrm{eV}$, whose localization clearly changes from edge to center and vice versa. Some bands are strongly localized at the edges, like those between 1 and $1.5 \mathrm{eV}$ near the $\mathrm{X}$ point. This can be a signal of an avoided crossing between these bands and bands around the Fermi level.

\section{APPENDIX B: ELECTRONIC BAND STRUCTURE AS A FUNCTION OF NANORIBBON WIDTH}

In this section we illustrate the influence of the ribbon width on the band structure. In the top panel of Fig. 10 we show the band structures of the 5-ZSiNR and 6-ZSiNR with the AFM triangle-pentagon edge reconstruction in comparison with the 4-ZSiNR one.

The ribbon width does not affect considerably the global band shape around the Fermi level, where the contribution of the edges is especially significant, so that the ribbons are still magnetic semiconductors. We notice some differences in the shape of the higher conduction bands and in the energy difference between the lower valence bands. The indirect gap between the VBM and the conduction band minimum at the $\Gamma$ point remains constant, while there is a tendency towards enlarging the direct band gap at the $\Gamma$ point and decreasing the direct band gap at VBM. Considering also that excitonic effects are more relevant in confined systems, these behaviors can result in a shift towards a higher energy of the main absorption peak in optical spectroscopy. The VBM shifts its position in the BZ, tending towards the $\mathrm{X}$ 

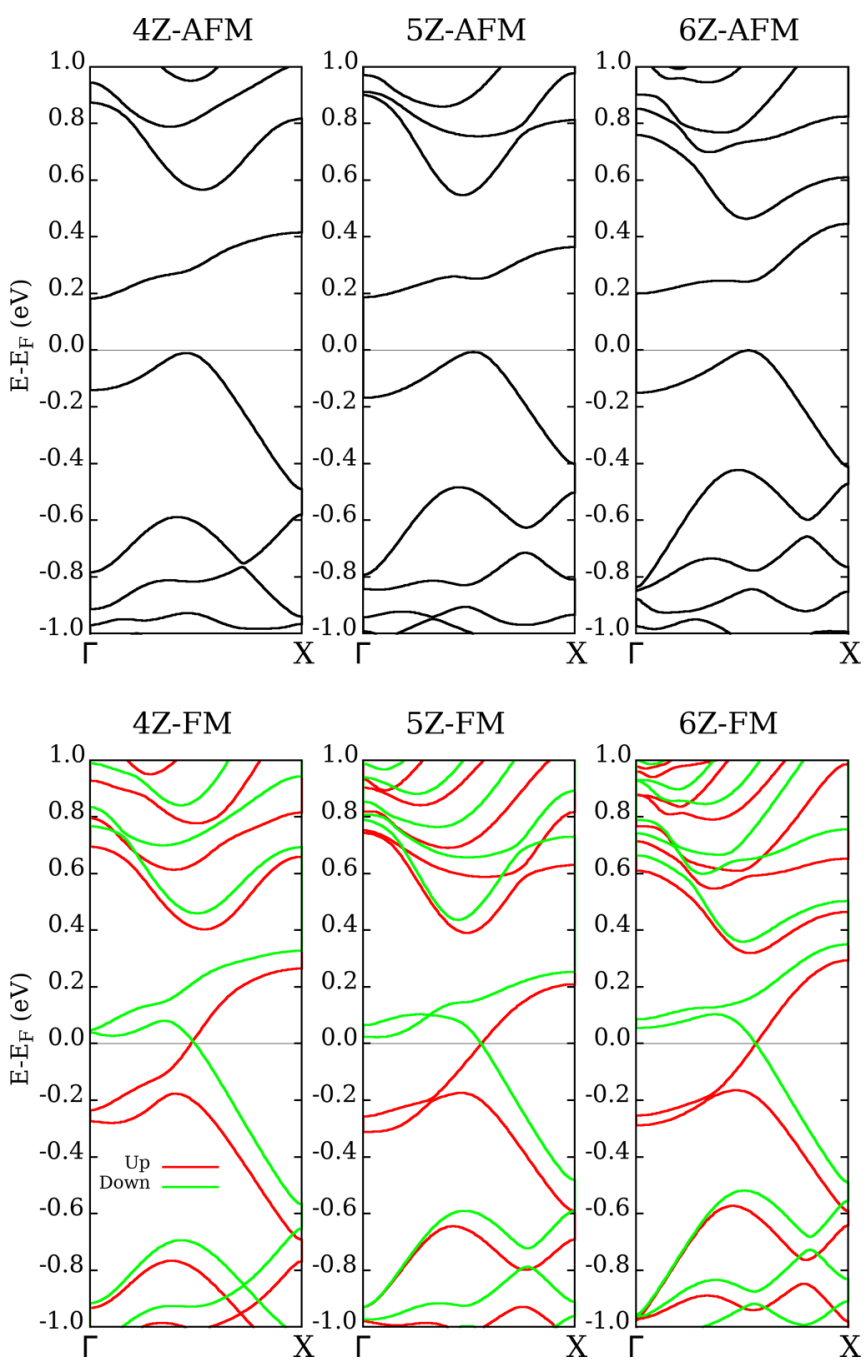

FIG. 10. Band structure for AFM (left panel) and FM (right panel) magnetic configurations for 4-, 5-, 6-chain zigzag silicene nanoribbons (with the triangle-pentagon edge reconstruction).

point. We notice also that the density of bands increases with width, consistently with the larger size of the system. These results are summarized in Table I. A PDOS analysis for the
TABLE I. Position of the VBM and energy gaps in AFM N-ZSiNRs.

\begin{tabular}{lccc}
\hline \hline & 4-ZSiNR & 5-ZSiNR & 6-ZSiNR \\
\hline VBM position $\left(\frac{2 \pi}{a}\right.$ units $)$ & 0.230 & 0.260 & 0.265 \\
Indirect energy gap $(\mathrm{eV})$ & 0.194 & 0.193 & 0.200 \\
$\Gamma$ direct energy gap $(\mathrm{eV})$ & 0.323 & 0.355 & 0.350 \\
VBM direct energy gap $(\mathrm{eV})$ & 0.293 & 0.259 & 0.243 \\
\hline \hline
\end{tabular}

6-ZSiNR [31] (not shown) demonstrates that the conduction is still due to the electrons localized on edge atoms. The intermediate and the central chains poorly contribute to the conduction.

We now analyze the ferromagnetic case. The band structures of the FM ZSiNRs are shown in the bottom panel of Fig. 10. Similarly to the VBM shift in the AFM structure, we notice a shift of the crossing point of the bands towards the $\mathrm{X}$ point of the BZ. This shift increases the total magnetization of the ribbon as reported in Table II, since there are more occupied spin-up states under the Fermi level. Since the edges are less interacting as the ribbon width increases, we notice also that the splitting between the two spin-up bands just below the Fermi level in the first half BZ reduces. The two spin-down bands just above the Fermi level show the same behavior. This can explain the degeneracy of these two bands found in Ref. [21] for the NM state of the 8-ZSiNR. In summary, we can say that the number of chains does not modify substantially the electronic properties of the nanoribbon, which maintains its semiconducting character in the AFM configuration and the semimetallic character in the FM one. Therefore it is possible to engineer the width depending on the purposes, without losing the desired conduction properties.

TABLE II. Position of the crossing points and the total magnetization in FM N-ZSiNRs.

\begin{tabular}{lccc}
\hline \hline & 4-ZSiNR & 5-ZSiNR & 6-ZSiNR \\
\hline Crossing point $\left(\frac{2 \pi}{a}\right.$ units $)$ & 0.244 & 0.277 & 0.283 \\
Total magnetization $\left(\mu_{b} /\right.$ cell $)$ & 0.97 & 1.093 & 1.16 \\
\hline \hline
\end{tabular}

[1] K. S. Novoselov, A. K. Geim, S. V. Morozov, D. Jiang, Y. Zhang, S. V. Dubonos, I. V. Grigorieva, and A. A. Firsov, Science 306, 666 (2004).

[2] A. Dimoulas, Microelectron. Eng. 131, 68 (2015).

[3] K. Takeda and K. Shiraishi, Phys. Rev. B 50, 14916 (1994).

[4] S. Cahangirov, M. Topsakal, E. Aktürk, H. Sahin, and S. Ciraci, Phys. Rev. Lett. 102, 236804 (2009).

[5] A. Debernardi and L. Marchetti, Phys. Rev. B 93, 245426 (2016).

[6] Y. Du, J. Zhuang, J. Wang, Z. Li, H. Liu, J. Zhao, X. Xu, H. Feng, L. Chen, K. Wu, X. Wang, and S. X. Dou, Sci. Adv. 2, e1600067 (2016).

[7] P. Vogt, P. De Padova, C. Quaresima, J. Avila, E. Frantzeskakis, M. C. Asensio, A. Resta, B. Ealet, and G. Le Lay, Phys. Rev. Lett. 108, 155501 (2012).
[8] H. Enriquez, A. Kara, A. J. Mayne, G. Dujardin, H. Jamgotchian, B. Aufray, and H. Oughaddou, J. Phys.: Conf. Ser. 491, 012004 (2014).

[9] A. Fleurence, R. Friedlein, T. Ozaki, H. Kawai, Y. Wang, and Y. Yamada-Takamura, Phys. Rev. Lett. 108, 245501 (2012).

[10] L. Meng, Y. Wang, L. Zhang, S. Du, R. Wu, L. Li, Y. Zhang, G. Li, H. Zhou, W. A. Hofer, and H. J. Gao, Nano Lett. 13, 685 (2013).

[11] S. Cahangirov, M. Audiffred, P. Tang, A. Iacomino, W. Duan, G. Merino, and A. Rubio, Phys. Rev. B 88, 035432 (2013).

[12] P. M. Sheverdyaeva, S. Kr. Mahatha, P. Moras, L. Petaccia, G. Fratesi, G. Onida, and C. Carbone, ACS Nano 11, 975 (2017). 
[13] E. Cinquanta, G. Fratesi, S. dal Conte, C. Grazianetti, F. Scotognella, S. Stagira, C. Vozzi, G. Onida, and A. Molle, Phys. Rev. B 92, 165427 (2015).

[14] C. Grazianetti, E. Cinquanta, and A. Molle, 2D Mater. 3, 012001 (2016).

[15] L. Tau, E. Cinquanta, D. Chiappe, C. Grazianetti, M. Fanciulli, M. Dubey, A. Molle, and D. Akinwande, Nat. Nanotechnol. 10, 227 (2015).

[16] L. C. Lew, Yan Voon, E. Sandberg, R. S. Aga, and A. A. Farajian, Appl. Phys. Lett. 97, 163114 (2010).

[17] Y. Ding and Y. Wang, Appl. Phys. Lett. 104, 083111 (2014).

[18] P. De Padova, C. Quaresima, P. Perfetti, B. Olivieri, C. Leandri, B. Aufray, S. Vizzini, and G. Le Lay, Nano Lett. 8, 271 (2008).

[19] P. De Padova, P. Perfetti, B. Olivieri, C. Quaresima, C. Ottaviani, and G. Le Lay, J. Phys.: Condens. Matter 24, 223001 (2012).

[20] S. Colonna, G. Serrano, P. Gori, A. Cricenti, and F. Ronci, J. Phys.: Condens. Matter 25, 315301 (2013).

[21] R. Li, J. Zhou, Y. Han, J. Dong, and Y. Kawazoe, J. Chem. Phys. 139, 104703 (2013).

[22] T. Xie, R. Wang, S. Wang, and X. Wu, Appl. Surf. Sci. 383, 310 (2016).

[23] C. Hogan, S. Colonna, R. Flammini, A. Cricenti, and F. Ronci, Phys. Rev. B 92, 115439 (2015).

[24] P. Giannozzi, S. Baroni, N. Bonini, M. Calandra, R. Car, C. Cavazzoni, D. Ceresoli, G. L. Chiarotti, M. Cococcioni, I. Dabo, A. Dal Corso, S. Fabris, G. Fratesi, S. de Gironcoli, R. Gebauer, U. Gerstmann, C. Gougoussis, A. Kokalj, M. Lazzeri, L. Martin-Samos, N. Marzari, F. Mauri, R. Mazzarello, S. Paolini, A. Pasquarello, L. Paulatto, C. Sbraccia, S. Scandolo, G. Sclauzero, A. P. Seitsonen, A. Smogunov, P. Umari, and R. M. Wentzcovitch, J. Phys.: Condens. Matter 21, 395502 (2009).

[25] M. Ferri, G. Fratesi, G. Onida, and A. Debernardi, Micro and Nano Engineering 1, 37 (2018).

[26] J. P. Perdew, K. Burke, and M. Ernzerhof, Phys. Rev. Lett. 77, 3865 (1996).

[27] H. J. Monkhorst and J. D. Pack, Phys. Rev. B 13, 5188 (1976).

[28] C. G. Broyden, IMA J. Appl. Math. 6, 76 (1970).
[29] A. Marini, C. Hogan, M. Grüning, and D. Varsano, Comput. Phys. Commun. 180, 1392 (2009).

[30] Y. W. Son, M. L. Cohen, and S. G. Louie, Nature (London) 444, 347 (2006).

[31] Matteo Ferri, Ab initio study of structural, electronic, and optical properties of silicene nanoribbons, M.A. thesis, Università degli Studi di Milano, 2016, http://www.yambo-code.org/ papers/Thesis_Matteo_Ferri.pdf.

[32] K. G. Sandeman, G. G. Lonzarich, and A. J. Schofield, Phys. Rev. Lett. 90, 167005 (2003).

[33] A. W. Joshi, Elements of Group Theory for Physicists (New Age International, 1997).

[34] F. Aryasetiawan and O. Gunnarsson, Rep. Prog. Phys. 61, 237 (1998).

[35] G. Onida, L. Reining, and A. Rubio, Rev. Mod. Phys. 74, 601 (2002).

[36] G. Strinati, Riv. Nuovo Cimento Soc. Ital. Fis. 11, 1 (1988).

[37] M. Bruno, M. Palummo, A. Marini, R. Del Sole, V. Olevano, A. N. Kholod, and S. Ossicini, Phys. Rev. B 72, 153310 (2005).

[38] D. Prezzi, D. Varsano, A. Ruini, A. Marini, and E. Molinari, Phys. Rev. B 77, 041404(R) (2008).

[39] We determine the $\omega_{D}$ as the frequency at which the real part of the dielectric function of 4-ZSiNR (computed without Drude term) vanishes. We checked that the behaviors of dielectric functions obtained by adding the Drude term do not depend on an accurate choice of the plasma frequencies (the dielectric function computed by using $\omega_{D}=4.3 \mathrm{eV}$ for the Drude term gives very similar results).

[40] As shown in Sec. IV, the system presents metallic states only at the edges of the nanoribbons, thus forming a $1 \mathrm{D}$ metallic electron system. It is known that the $1 \mathrm{D}$ free-electron system presents peculiar dielectric properties and the total suppression of the electron-hole single-particle excitation continua at low energy [41]. As a consequence of 1D effects, the inclusion of the electron-hole interaction in the BSE spectrum can produce the vanishing of the imaginary part of the dielectric function as the frequency tends to zero.

[41] S. Das Sarma and E. H. Hwang, Phys. Rev. B 54, 1936 (1996).

[42] W. Wei, Y. Dai, B. Huanga, and T. Jacob, Phys. Chem. Chem. Phys. 15, 8789 (2013). 